\title{
An Improved Staining Method for Low Signal LacZ Reporter Detection in Mouse Embryos
}

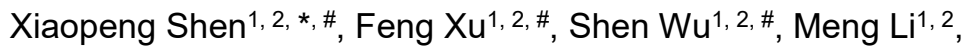 \\ Jingyi Zhang ${ }^{1,2}$, Rui Liang ${ }^{3}$ and Yu Liü, *
}

\begin{abstract}
${ }^{1}$ School of Life Sciences, Anhui Normal University, Wuhu, China; ${ }^{2}$ The Key Laboratory of Biomedicine in Gene Diseases and Health of Anhui Higher Education Institutes, Anhui Normal University, Wuhu, China; ${ }^{3}$ Department of Biology and Biochemistry, University of Houston, Houston, United States of America

*For correspondence: shenxiaopeng cn@163.com; yliu54@uh.edu

\#Contributed equally to this work
\end{abstract}

\begin{abstract}
[Abstract] In many fields of biology, especially in the field of developmental biology, LacZ reporter staining is an approach used to monitor gene expression patterns. In the LacZ reporter system, the LacZ gene is inserted in the endogenous location of the target gene via gene knock-in or by constructing a transgenic cassette in which $L a c Z$ is placed downstream of the promoter of the target gene being examined. Currently, the most common LacZ staining methods used are X-gal/FeCN staining and Sgal/TNBT staining. A serious limitation of both of these methods is that they are not effective when the LacZ gene is expressed at a low level. In an attempt to remedy this problem, we have established a new staining protocol which combines both methods. When compared to them, the method described here is better for visualizing lowly expressed genes and it has low background with high sensitivity.
\end{abstract}

Keywords: LacZ reporter, Mouse embryo, Low expression, Low background, High sensitivity

[Background] The $L a c Z$ gene has been widely used as a reporter gene for detecting gene expression patterns (Stevens et al., 1989; Bonnerot and Nicolas, 1993). The protein encoded by the LacZ gene is $\beta$-galactosidase, which is able to produce visible color when incubated with specific substrates. Usually, $L a c Z$ is placed downstream of a target gene promoter in lieu of the coding sequence of the gene. Therefore, visualizing $L a c Z$ expression patterns models the endogenous expression pattern of the target gene (Figure 1A). The most popular LacZ staining approach is the $X-g a l / F e C N$ method, in which $\beta$ galactosidase catalyzes X-gal (5-bromo-4-chloro-3-indolyl- $\beta$-D-galactopyranoside) hydrolysis into 5bromo-4-chloro-3-hydroxyindole and galactose. Then, 5-bromo-4-chloro-3-hydroxyindole is oxidized into an insoluble blue dimer with the help of potassium ferricyanide and potassium ferrocyanide, which finally displays blue color (Figure 1B) (Pearson et al., 1963; Lojda, 1970). While the X-gal/FeCN staining method is highly specific and has low background, it is unable to detect genes expressed at low levels due to the low sensitivity of this stain (Gugliotta et al., 1992; Sundararajan et al., 2012). The S-gal/TNBT staining method was developed to study genes with low expression. S-gal (6-Chloro-3-indolyl- $\beta$-Dgalactopyranoside), a chromogenic substrate like X-gal, can be hydrolyzed by $\beta$-galactosidase and TNBT (Tetranitro Blue Tetrazolium) to produce dark-brown formazan compounds under reducing 
conditions (Figure 1C). The S-gal/TNBT method is more sensitive than the $\mathrm{X}$-gal/FeCN one, but a drawback is that it can have strong non-specific background staining (Sundararajan et al., 2012).

By combining both the methods described above, we developed an improved LacZ reporter staining protocol that is highly sensitive and highly specific. This method adds an additional staining step from the S-gal/TNBT method to the X-gal/FeCN method. In our staining technique, the first staining step, which is from the $\mathrm{X}$-gal/FeCN method, creates an oxidative environment which consumes non-specific enzymatic activity; the second step, which is from the S-gal/TNBT method, is a reaction that is specific for $\beta$-galactosidase and results in a sensitive and specific signal. The improved method described here has been validated by detecting several genes in different embryo stages. Furthermore, this method has been used to study relatively highly expressed genes with good results: strong and specific staining, with slightly higher background than the X-gal/FeCN method (Shen et al., 2017).

A

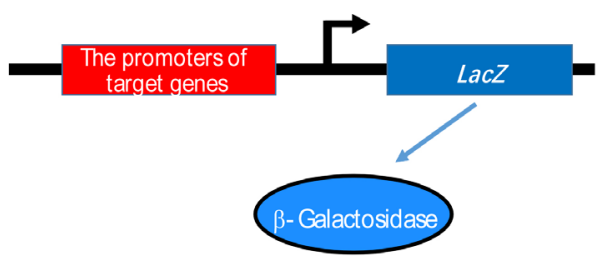

B

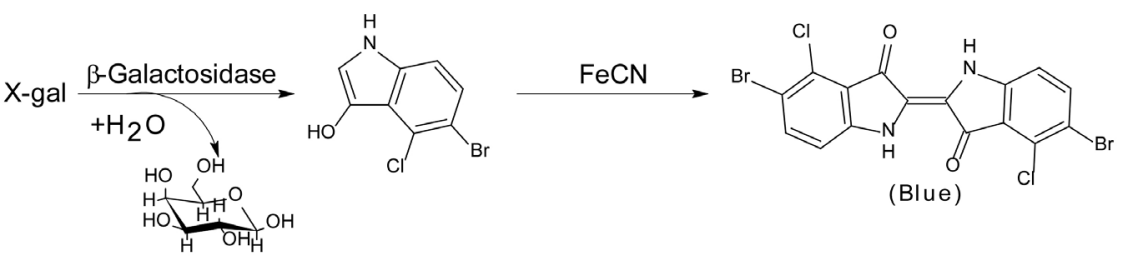

C

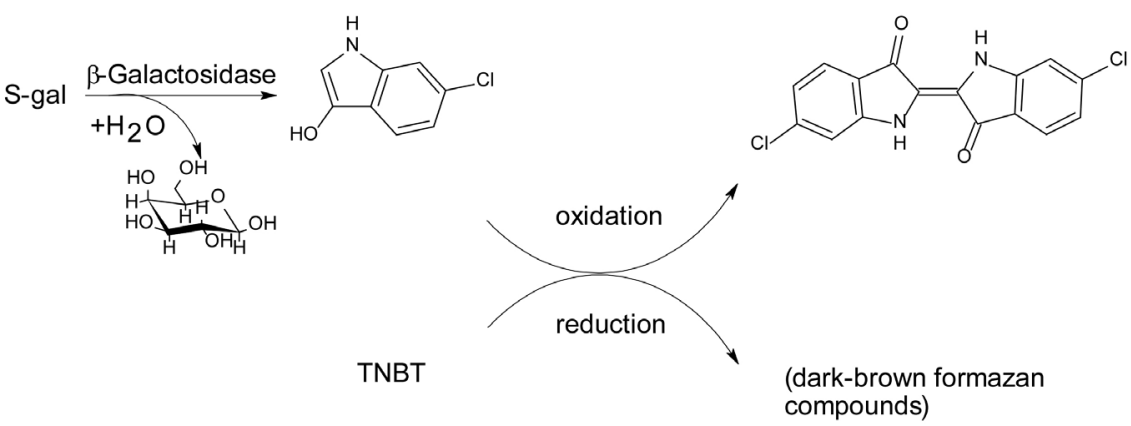

Figure 1. Schematic diagram of LacZ reporters and -Galactosidase reactions. (A) The LacZ reporter gene is placed downstream of the target gene's promoter. Thus, when the expression of the target gene is induced, the gene product of $L a c Z$, $\beta$-galactosidase, is produced. The key steps of the enzymatic reactions in the X-gal/FeCN (B) and S-gal/TNBT (C) staining methods. 


\section{Materials and Reagents}

1. Sterile DNase/RNase free pipette tips $10 \mu \mathrm{l}, 200 \mu \mathrm{l}, 1,000 \mu \mathrm{l}$ (Labselect, catalog numbers: T001-10, T-001-200, T-001-1000)

2. 6-well plates (NUNC, catalog number: 140675)

3. Embryos collected from pregnant mice at E8.5, E9.5, and E10.5 stages. The mouse strain used here has been described previously in (Shen et al., 2017)

4. Paraformaldehyde (PFA) (Sigma, catalog number: P6148)

5. Nonidet P-40 (NP-40) (Sangon Biotech, catalog number: A510110)

6. Sodium deoxycholate (Sigma, catalog number: D6750)

7. X-gal (Gold Bio, catalog number: X4281C)

8. $\mathrm{K}_{3} \mathrm{Fe}(\mathrm{CN})_{6}$ (Sigma, catalog number: 244023)

9. $\mathrm{K}_{4} \mathrm{Fe}(\mathrm{CN})_{6}$ (Sigma, catalog number: P3289)

10. $\mathrm{MgCl}_{2}$ (Sigma, catalog number: M8266)

11. EGTA (Sigma, catalog number: E3889)

12. IGEPAL (Sigma, catalog number: I8896)

13. Salmon-gal (S-gal) (Lab Scientific, catalog number: X668)

14. Tetranitro Blue Tetrazolium (TNBT) (VWR, catalog number: TCT0250)

15. $\mathrm{NaCl}$ (Sigma, catalog number: S7653)

16. $\mathrm{KCl}$ (Sigma, catalog number: P9333)

17. $\mathrm{Na}_{2} \mathrm{HPO}_{4}$ (Sigma, catalog number: $\mathrm{S} 7907$ )

18. $\mathrm{KH}_{2} \mathrm{PO}_{4}$ (Sigma, catalog number: $\mathrm{P} 5655$ )

19. $\mathrm{NaH}_{2} \mathrm{PO}_{4}$ (Sigma, catalog number: S9638)

20. PBS (see Recipes)

21. 4\% PFA (see Recipes)

22. $0.1 \mathrm{M}$ phosphate buffer ( $\mathrm{pH}$ 7.3) (see Recipes)

23. Wash buffer (see Recipes)

24. Staining buffer 1 (see Recipes)

25. Staining buffer 2 (see Recipes)

\section{Equipment}

1. Pipettes (Gilson, catalog numbers: F144801, F123600, F144058M, F123602)

2. Humidified chambers (for example, covered ice boxes or foam boxes with wet paper tower)

3. Dissecting forceps (Fine Science Tools, model: Dumont \#5)

4. Stereomicroscope (Leica, model: MZ12.5)

5. Orbital Shaker (MIULAB, GS-20)

6. Microbiological Incubator (Thermo Scientific, PR305225G)

7. Microscope (Leica, DFC340 FX digital FireWire Camera System) 


\section{Software}

1. Leica Application Suite V3.7 (Image taking software for Leica DFC340 FX digital FireWire Camera System)

\section{Procedure}

1. Collect embryos from pregnant mice as reported previously (Shea and Geijsen, 2007) and fix the embryos in $10 \mathrm{ml}$ 4\% PFA (Recipe 2) in 6-well plates at room temperature (the volumes used here and in the following steps are for one embryo). The 6-well plates should be placed in humidified chambers, which are kept on orbital shakers set to $65 \mathrm{rpm}$. The fixation time varies and depends on the developmental stage of the embryos. The following table lists the recommended fixation times.

\begin{tabular}{|l|l|l|l|l|}
\hline Embryo stages & E7.5 & E8.5 & E9.5 & E10.5 \\
\hline Fixation time (min) & 10 & 15 & 20 & 30 \\
\hline
\end{tabular}

2. Use forceps to transfer the embryos to a new well of the 6-well plate filled with $10 \mathrm{ml}$ Wash Buffer (Recipe 4). Wash the embryos three times with a $10 \mathrm{~min}$ incubation at room temperature for each wash. During the washing steps, the 6-well plates should be placed in humidified chambers on an orbital shaker set at $65 \mathrm{rpm}$.

3. Use forceps to transfer the embryos to a new well of the 6-well plate filled with $10 \mathrm{ml}$ Staining Buffer 1 (Recipe 5). Incubate overnight at $37^{\circ} \mathrm{C}$ in a microbiological incubator (remember to protect samples from light). The 6-well plates should be placed in humidified chambers.

4. Use forceps to transfer the embryos to a new well of the 6-well plate filled with $10 \mathrm{ml}$ Wash Buffer and incubate for $10 \mathrm{~min}$ at room temperature. Next use forceps to transfer the embryos to a new well of the 6-well plate with Staining Buffer 2 (Recipe 6) and incubate at $37^{\circ} \mathrm{C}$ in a microbiological incubator. The 6-well plates should be placed in humidified chambers. The staining should be closely monitored with a stereomicroscope until specific staining appears. The staining time could vary from several minutes to one hour.

5. Use forceps to transfer the embryos to a new well of the 6-well plate filled with $10 \mathrm{ml}$ Wash Buffer and wash three times. Incubate each wash for $10 \mathrm{~min}$. During the wash, the 6-well plates should be placed in humidified chambers, on an orbital shaker set to $65 \mathrm{rpm}$. After the embryos are washed, they can be imaged directly or kept at $4{ }^{\circ} \mathrm{C}$ in Wash Buffer for up to one week.

6. Take images using Leica Application Suite V3.7 on a Leica DFC340 FX microscope. We use the Z-stack option in the Leica Application Suite V3.7 because the stained embryos are relatively thick. The images generated from the Z-stack processing are used as final results. Embryos that do not express the $L a c Z$ gene are used as negative controls. Figure 2 shows representative staining images of $\mathrm{X}$-gal/FeCN, S-gal/TNBT, and the improved method described here. 
A

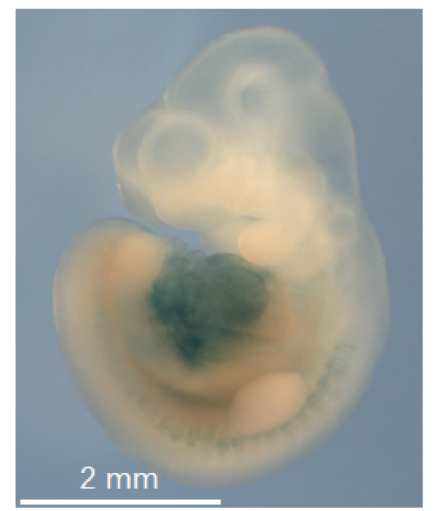

B

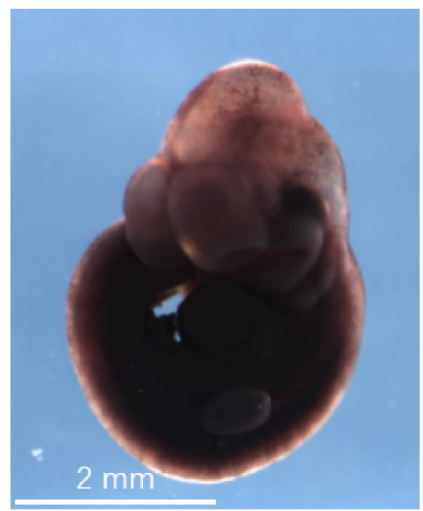

C

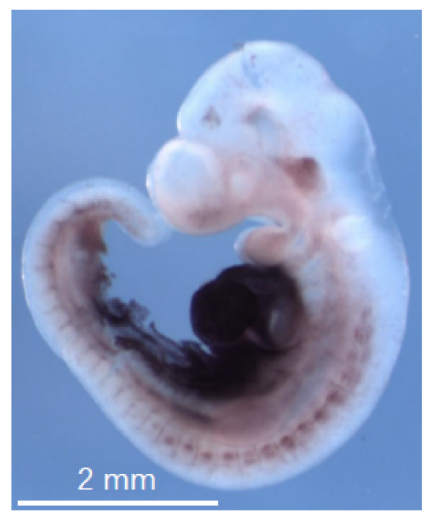

Figure 2. The representative LacZ staining images of $X-g a l / F e C N, S-g a l / T N B T$, and the improved method in detecting miR-322/-503's expression in E10.5 embryos. A. Embryos stained with X-gal/FeCN method. B. Embryos stained with S-gal/TNBT method. C. Embryos stained with the improved method described here. The images were taken with a Leica DFC340 FX microscope at 10x magnification.

\section{Data analysis}

Scale bars, annotations, and arrows that point at the specific stained regions are added to the embryo images.

\section{$\underline{\text { Recipes }}$}

1. PBS

$137 \mathrm{mM} \mathrm{NaCl}$

$2.7 \mathrm{mM} \mathrm{KCl}$

$10 \mathrm{mM} \mathrm{Na}_{2} \mathrm{HPO}_{4}$

$1.8 \mathrm{mM} \mathrm{KH}_{2} \mathrm{PO}_{4}$

in distilled water, $\mathrm{pH}=7.4$

2. $4 \%$ PFA

Dissolve 4\% PFA in PBS

Heat at $50{ }^{\circ} \mathrm{C}$ and vortex until fully dissolved

Then adjust the $\mathrm{pH}$ to $7.2 \sim 7.4$

3. $0.1 \mathrm{M}$ phosphate buffer $(\mathrm{pH} 7.3)$

$23 \% \mathrm{NaH}_{2} \mathrm{PO}_{4}$ solution (0.2 $\mathrm{M} \mathrm{NaH}_{2} \mathrm{PO}_{4}$ in distilled water)

$77 \% \mathrm{Na}_{2} \mathrm{HPO}_{4}$ solution (0.2 $\mathrm{M} \mathrm{Na}_{2} \mathrm{HPO}_{4}$ in distilled water)

4. Wash buffer

$0.02 \%$ NP-40

$0.01 \%$ sodium deoxycholate in PBS 
5. Staining buffer 1

$5 \mathrm{mM} \mathrm{K}_{3} \mathrm{Fe}(\mathrm{CN})_{6}$

$5 \mathrm{mM} \mathrm{K}_{4} \mathrm{Fe}(\mathrm{CN})_{6}$

$0.02 \% \mathrm{NP}-40$

$0.01 \%$ deoxycholate

$2 \mathrm{mM} \mathrm{MgCl} 2$

5 mM EGTA

$1 \mathrm{mg} / \mathrm{ml} \mathrm{X-gal} \mathrm{in} \mathrm{PBS}$

6. Staining buffer 2

$1 \mathrm{mg} / \mathrm{ml} \mathrm{S-gal}$

$0.4 \mathrm{mM}$ TNBT

$0.1 \%$ sodium deoxycholate

$0.2 \%$ IGEPAL

$2 \mathrm{mM} \mathrm{MgCl} 2$ in $0.1 \mathrm{M}$ phosphate buffer ( $\mathrm{pH} 7.3)$

\section{Acknowledgments}

We thank Wei Yu at the University of Houston for guidance in animal operations; Wenjing Bao at the Liaoning University of Traditional Chinese Medicine for technical support. This work was supported by the National Natural Science Foundation of China (No. 31701289), Anhui Provincial Natural Science Foundation (No. 1808085QH234), Educational Commission of Anhui Province of China (No. KJ2017A319), Foundation for High-level Talents in Higher Education of Anhui Province of China and Start-up Funds from the Anhui Normal University.

\section{Competing interests}

No competing interests exist.

\section{Ethics}

All animal-related work has been approved by the Institutional Animal Care and Use Committee (IACUC).

\section{$\underline{\text { References }}$}

1. Bonnerot, C. and Nicolas, J. F. (1993). Application of LacZ gene fusions to postimplantation development. Methods Enzymol 225: 451-469.

2. Gugliotta, P., Pacchioni, D. and Bussolati, G. (1992). Staining reaction for $\beta$-galactosidase in immunocytochemistry and in situ hybridization. Eur J Histochem 36(2): 143-148. 
Please cite this article as: Shen et. al., (2019). An Improved Staining Method for Low Signal LacZ Reporter Detection in Mouse Embryos, Bio-protocol 9

3. Lojda, Z. (1970). Indigogenic methods for glycosidases. I. An improved method for $\beta$-Dglucosidase and its application to localization studies on intestinal and renal enzymes. Histochemie 22(4): 347-361.

4. Pearson, B., Wolf, P. L. and Vazquez, J. (1963). A comparative study of a series of new indolyl compounds to localize $\beta$-galactosidase in tissues. Lab Invest 12: 1249-1259.

5. Shea, K. and Geijsen, N. (2007). Dissection of 6.5 dpc mouse embryos. J Vis Exp (2): 160.

6. Shen, X., Bao, W., Yu, W., Liang, R., Nguyen, B. and Liu, Y. (2017). An improved method with high sensitivity and low background in detecting low $\beta$-galactosidase expression in mouse embryos. PLoS One 12(5): e0176915.

7. Stevens, M. E., Meneses, J. J. and Pedersen, R. A. (1989). Expression of a mouse metallothionein-Escherichia coli $\beta$-galactosidase fusion gene (MT- $\beta$ gal) in early mouse embryos. Exp Cell Res 183(2): 319-325.

8. Sundararajan, S., Wakamiya, M., Behringer, R. R. and Rivera-Perez, J. A. (2012). A fast and sensitive alternative for $\beta$-galactosidase detection in mouse embryos. Development 139(23): 4484-4490. 